\title{
ESCALA DIFICULDADES NA AMAMENTAÇÃo
}

\author{
Emília Coutinho \\ Escola Superior de Saúde, Instituto Politécnico de Viseu, UICISA: E - IPV/ESSV \\ ecoutinhoessv@gmail.com \\ Paula Nelas \\ Escola Superior de Saúde, Instituto Politécnico de Viseu, UICISA: E - IPV/ESSV \\ pnelas@gmail.com \\ Cláudia Chaves \\ Escola Superior de Saúde, Instituto Politécnico de Viseu, CI\&DEI \\ claudiachaves21@gmail.com
}

Recepción Artículo: 22 octubre 2021 Admisión Evaluación: 22 octubre 2021 Informe Evaluador 1: 23 octubre 2021 Informe Evaluador 2: 24 octubre 2021 Aprobación Publicación: 24 octubre 2021

\section{RESUMO}

Enquadramento: Muitas mães experimentam dificuldades na amamentação, especificamente no início do processo da amamentação. 0 apoio adequado de profissionais de saúde treinados, nomeadamente os enfermeiros, é crucial para minimizar o impacto dos problemas da amamentação. Objetivo: Avaliar a estrutura interna da escala de dificuldades de amamentação através da análise factorial exploratória e confirmar o modelo fatorial hipotetizado através da analise factorial confirmatória. Participantes: Este estudo realizou-se com uma amostra não probabilística por conveniência constituída por 674 mulheres com idades compreendidas entre os 15 e 49 anos (média $=31,65$ anos $\pm 5,95)$ a coabitar com companheiro (93,6\%). São de nacionalidade portuguesa (97,0\%), possuem habilitações literárias superiores ao $12^{\mathrm{a}}$ ano $47,9 \%$ e encontram-se empregadas $84,3 \%$ da totalidade da amostra. Método: Estudo metodológico, em que a escala foi testada através da validade aparente de conteúdo e constructo. As propriedades psicométricas, foram avaliadas com recurso à consistência interna pelo coeficiente Alpha ( ) de Cronbach e validade de construto através da validade factorial exploratória e confirmatória, validade convergente e validade discriminante. Resultados: A análise factorial exploratória levou à obtenção de uma estrutura trifatorial que explicava no total $68,64 \%$ da variância e com um coeficiente alfa de Cronbach de 0,919. Com a análise fatorial confirmatória foram eliminados dois itens por apresentarem problemas de multicolinearidade. Os índices gerais de bondade de ajustamento global são fiáveis, confirmando a qualidade do ajuste do modelo aos dados empíricos. (x2/gl= 3,892; GFI=0,962; $\mathrm{CFI}=0,974 ; \mathrm{RMR}=0,049 ; \mathrm{RMSEA}=0,065$ e SRMR $=0,042$ ). Conclusões: Os resultados evidenciam que a escala apresenta uma estrutura fatorial com resultados satisfatórios de validade e de confiabilidade, representando adequadamente os constructos em ques- 


\section{ESCALA DIFICULDADES NA AMAMENTAÇÃo}

tão. A escala de dificuldades de amamentação pode ser utilizada como instrumento de pesquisa para avaliar as dificuldades sentidas pelas puérperas no processo de amamentação.

Palavras chave: aleitamento materno; problemas; maternidade; enfermagem; puérpera

\section{ABSTRACT}

Breastfeeding difficulties scale. Background: Many mothers experience breastfeeding difficulties, specifically early in the breastfeeding process. Adequate support from trained health workers, notably nurses, is crucial to minimise the impact of breastfeeding problems. Objectives To evaluate the internal structure of the breastfeeding difficulties scale through exploratory factor analysis and confirm the hypothesised factor model through confirmatory factor analysis. Participants: The data used were obtained by applying them to a nonprobabilistic convenience sample consisting of 674 women aged between 15 and 49 years (average $=31,65$ years $\pm 5,95)$, the majority living with a partner $(93,6 \%)$. They are of Portuguese nationality $97,0 \%$, have an academic level above the 12 th grade $47,9 \%$ and are employed $84,3 \%$ of the total sample. Method: This is a methodological study, in which the scale was tested through content and construct validity. The psychometric properties were assessed using Cronbach's Alpha coefficient ( ) for internal consistency and construct validity through exploratory and confirmatory factorial validity, convergent validity, and discriminant validit. Results: The exploratory factorial analysis led to a three-factor structure which explained a total of $68,64 \%$ of the variance and had a Cronbach's alpha coefficient of 0,919 . With the confirmatory factor analysis, two items were eliminated for presenting multicollinearity problems. The overall goodness of fit indices are reliable, confirming the quality of the model's fit to the empirical data. ( $x 2 / \mathrm{gl}=3,892 ; \mathrm{GFI}=0,962 ; \mathrm{CFI}=0,974 ; \mathrm{RMR}=0,049 ; \mathrm{RMSEA}=0,065$ and $\mathrm{SRMR}=0,042$ ).

Conclusion: The results show that the breastfeeding difficulties scale has a factor structure with satisfactory results of validity and reliability, adequately representing the constructs in question. The breastfeeding difficulties scale can be used as a research tool to assess the difficulties experienced by postpartum women in the breastfeeding process.

Keywords: breastfeeding; problems; maternity; nursing; puerperal

\section{INTRODUÇÃO}

A amamentação é um importante contributo para a sobrevivência, saúde, bem-estar e qualidade de vida das populações em todo o mundo. Constitui-se como uma poderosa linha de defesa contra todas as formas de desnutrição infantil, incluindo magreza extrema e obesidade, para além de atuar como a primeira vacina dos recémnascidos, protegendo-os contra muitas doenças infantis comuns. Nesse sentido, a OMS conjuntamente com a Unicef recomenda que 0 aleitamento materno se deve iniciar na primeira hora de vida, ser exclusivo até aos 6 meses de vida e a sua manutenção, com alimentos complementares, deve continuar pelo menos, até ao segundo ano de vida (WHO \& UNICEF, 2021).

Apesar dos benefícios do aleitamento materno (AM) serem reconhecidos mundialmente verifica-se no entanto, uma diminuição progressiva e acentuada nas taxas de prevalência do aleitamento materno, após a saída da maternidade (Oliveira, 2016), deixando transparecer dificuldades ao longo do processo de amamentação com os quais as mães/casais se confrontam e que se tornam grandes desafios à amamentação. Inumeros estudos apontam diversas barreiras ao aleitamento materno (Office of the Surgeon General (US), Centers for Disease Control and Prevention (US), Office on Women's Health (US), The Surgeon General's Call to Action to Support Breastfeeding. Rockville (MD), \& Office of the Surgeon General (US), 2011) como a falta de conhecimento nomeadamente dos riscos associados à falta da amamentação; normas sociais que consideram o aleitamento artificial como mais aceitável; pobreza e falta de suporte social em que as mães não se sentem apoiadas pela família e amigos para ultrapassar as dificuldades com a amamentação, ou têm também eles experiências negativas ou atitudes que desencorajam a amamentação; constrangimento em amamentar em público, quer por desconhecimento em o poder fazer, quer por serem advertidas para o não fazerem em algumas sociedades; problemas na lactação relacionados com má prática posicional ou de estimulação por parte do bebé, ou por não se sentirem 
apoiadas por parte dos profissionais de saúde para ultrapassar as dificuldades inerentes ao processo de amamentação; emprego e falta de condições para amamentar, com falta de flexibilidade de horários, privacidade ou até creches para deixarem as crianças durante o período laboral, ausência de licença à maternidade ou falta de condições para dela usufruírem; barreiras dos próprios serviços de saúde, quer pelas politicas hospitalares quanto pelas práticas clinicas, em que dão pouca prioridade ao apoio à amamentação e a campanhas que a promovam, com rotinas inadequadas à amamentação, que praticam a suplementação desnecessária, o recurso abusivo a partos por cesariana que dificultam o estabelecimento da amamentação, a própria experiencia negativa dos próprios profissionais que se sobrepõe em alguns casos aos seus conhecimentos científicos.

É assim importante conhecer as condições técnicas, maternas e do recém-nascido que se apresentam por parte das mães/casais de modo a facilitar uma prática dirigida às suas necessidades e preocupações (Rasteiro, Santos, \& Coutinho, 2021) de modo a que a amamentação bem sucedida seja uma realidade mundial.

\section{OBJETIVOS}

Avaliar a estrutura interna da escala de dificuldades de amamentação através da análise factorial exploratória e confirmar o modelo fatorial hipotetizado através da analise factorial confirmatória.

\section{AMOSTRA E PARTICIPANTES}

Este estudo foi realizado com uma amostra não probabilístico por conveniência constituída por 674 puérperas, com idades entre 15 e 49 anos ( $M=31,65$ anos; $d p=5,95)$. Globalmente a amostra $(97,0 \%)$ é de nacionalidade portuguesa e coabita (93,6\%) com companheiro. Na sua maioria as mães possuem entre $09^{0}$ ano $(29,6 \%)$ e habilitações superiores ou iguais ao $12^{\circ}$ ano $(49,8 \%)$. Cerca de $85,0 \%$ exercem uma profissão auferindo (42,9\%) um vencimento entre 500 e 1000 euros, e mais de metade (51,5\%) tem em média um filho. Habitam na cidade $68,8 \%$ e em $44,5 \%$ o agregado familiar é constituído por três pessoas.

Para a selecção da amostra foi realizado o levantamento através do programa SINUS de toda as mães que pariram há pelo menos dois anos e que não realizaram a consulta de saúde infantil da criança. Procedeu-se à elaboração de uma listagem com as mães passiveis de participarem no estudo. No dia da consulta dos dois anos, cada mãe foi abordada no sentido de participar no estudo. Foram dados a conhecer os objetivos do estudo e 0 interesse científico do mesmo. Foram informadas que a participação era voluntária podendo desistir em qualquer momento. Assegurou-se a confidencialidade

\section{PROCESSO NA CONSTRUÇÃO DA ESCALA}

Muitas das mães que experimentam dificuldades durante a amamentação, especialmente no início do processo da amamentação poderiam conseguir ultrapassar essas dificuldades se tivessem o apoio adequado de profissionais de saúde, nomeadamente enfermeiros, competentes (Walker, 2008). São várias as dificuldades que as mães enfrentam e que levam ao abandono precoce da amamentação, nomeadamente de condições técnica, de condições maternas e de condições inerente ao recém.

Foi com base nesses pressupostos que se construiu um instrumento de recolha de dados para avaliar as dificuldades de amamentação. Trata-se de uma escala tipo Likert inicialmente com 20 asserções e cada uma com cinco opções de resposta em que um (1) representa nenhuma dificuldade; dois (2) pouca dificuldade); três (3) alguma dificuldade; quatro (4) muita dificuldade; cinco (5) total dificuldade.

A sua construção baseou-se na fundamentação teórica realizada para o efeito e na experiência investigadores na área de construção de escalas. Definidos os constructos da escala, procurou-se para cada definir um conjunto de itens que permitissem medir o construto.

Realizou-se inicialmente a validade de conteúdo, usando-se para o efeito dois procedimentos: análise de juízes e analise semântica.

Assim, os itens da escala foram submetidos a uma consulta de experts na área do constructo no sentido de avaliar a sua pertinência no que respeita a aspectos considerados importantes para garantir a qualidade psico- 


\section{ESCALA DIFICULDADES NA AMAMENTAÇÃo}

métrica da escala. A quatro juízes da área da saúde materna foi-Ihes solicitado que julgassem os itens quanto á sua pertinência e à relação do item com a dimensão avaliada, para além de thes serem solicitado outras informações e sugestões para 0 aperfeiçoamento dos itens.

A etapa sequente consistiu na análise descritiva dos pareceres emitidas pelos juízes, verificando-se as frequências e percentagens de concordância com o constructo e com a pertinência do item com a dimensão avaliada. Definiu-se como critério incorporar os itens com concordância entre juízes acima de $75 \%$ Os itens com percentagem inferior foram eliminados ou quando sugerido sofreram modificações. Este procedimento levou à modificação de 2 itens e à eliminação de sete itens por não obterem concordância inter-juizes.

Por fim, com os itens remanescentes procedeu-se a uma análise semântica por forma a analisar a compreensão dos itens, tendo-se realizado um pré-teste com 10 mães. Todas as modificações e sugestões apresentadas foram incorporadas na versão final da escala.

\section{Procedimentos para análise de dados}

As propriedades métricas da escala avaliaram-se através de estudos de validade e fiabilidade. Os estudos de fiabilidade configuraram a determinação da homogeneidade dos itens através do coeficiente de (i) correlação de Pearson dos diversos itens a nota global assumindo-se como valores de referência correlações superiores a 0,20; (ii) Determinação do coeficiente alfa de Cronbach com os valores de referencia: > 0,9 muito boa; 0,8 - 0,9 boa; 0,7 - 0,8 média; 0,6-0,7 razoável; 0,5 - 0,6 Má; <0,5 inaceitável (Pestana e Gageiro, 2014)

Os estudos de validade compreenderam a analise factorial exploratória e a análise factorial confirmatória. $\mathrm{Na}$ análise factorial exploratória optou-se pelo método dos componentes principais e pelo uso da rotação ortogonal do tipo varimax. Para a retenção de factores consideraram-se os valores próprios (autovalores) superiores a $1 \mathrm{e}$ 0 gráfico de declive (scree plot). Como critério de saturação dos itens consideraram-se valores iguais ou superiores a 0,40. Em relação à análise fatorial confirmatória (AFC) foi usada a matriz de covariâncias e adotado 0 algoritmo da máxima verosimilhança MLE (Maximum-Likelihood Estimation) para estimação dos parâmetros.

Como procedimentos estatísticos foi considerado: (i) sensibilidade dos itens, avaliada pelos valores de assimetria (Sk) e achatamento (Ku), considerando-se como valores de referência $S K<=3$ e $K u<=7$; (ii) qualidade do ajustamento global do modelo factorial usando-se os índices: razão do qui quadrado vs graus de liberdade (x2/gl) são adequados valores inferiores a 5; googness-of-fit (GFI), e comparative fit index (CFI). Para estes índices são adequados valores superiores a 0,90 , root-square error of aproximation (RMSEA) standardized root mean square residual (SRMR) e root mean square residual (RMR), são aceites valores inferiores a 0,08; (iii) qualidade do ajustamento local efetuada pelos pesos fatoriais ( ) e pela fiabilidade individual dos itens $\left(r^{2}\right)$ com valores de $0,50 \mathrm{e}$ 0,25 respectivamente; (iv) fiabilidade compósita (FC) estima a consistência interna dos itens relativos ao fator. Uma FC $\geq 0,70$ é considerada apropriado; (v) validade do construto avaliada pela validade convergente (VEM) e validade discriminante. Valores de VEM $\geq 0,50$ indica validade adequada; (vi) validade discriminante dos factores (VD), considera-se que há evidência da VD quando a correlação ao quadrado entre os fatores é menor do que a VEM para cada fator.

0 ajustamento do modelo foi realizado a partir dos índices de modificação propostos pelo AMOS.

\section{Procedimentos éticos}

0 protocolo de pesquisa foi previamente submetido à Comissão Nacional de Proteção de Dados (CNPD) e à comissão de ética dos Hospitais e Centros de Saúde envolvidos no estudo, aos quais, garantimos o cumprimento dos princípios gerais de ética em investigação. A todas as participantes foram fornecidas as informações necessárias e solicitada a assinatura do termo de consentimento livre e esclarecido. Foi assegurado o seu anonimato e a confidencialidade dos dados e respeitada a sua autonomia. Foram ainda informadas de que a sua participação era totalmente livre, podendo desistir do estudo em qualquer momento e que por essa participação não usufruiriam de qualquer ganho/benefício nem incorreriam em nenhuma perda/malefício. 


\section{RESULTADOS ALCANÇADOS}

Iniciamos o estudo psicométrico determinando as estatísticas de cada um dos itens ea sensibilidade dos itens através da assimetria e curtose revelando os resultados que os mesmos se encontram dentro dos parâmetros de referência com valores de assimetria a oscilar em valores absolutos entre 0,422 e 1,052 e valores de curtose entre 0,060 e 0,646 e com um coeficiente multivariado de mardia de 1,521

Os índices médios e respetivos desvio padrão apresentam-se bem centrados, situando-se na sua totalidade abaixo do ponto médio o que significa que em média as mães revelam poucas dificuldades na amamentação.

Os coeficientes de correlação item total corrigido regista que todos os itens possuem correlações acima de 0,20 (Pestana e Gageiro, 2014) pelo que nenhum foi excluido. Analisando os coeficientes alfa de Cronbach, por item, observamos que são muito bons ao situarem-se acima de 0,909 com um alfa global de 0,919. 0 coeficiente de bipartição regista valores de alfa de Cronbach ligeiramente mais fracos ao referido, sendo que para a primeira metade 0 valor de alfa é de 0,895 e para a segunda de 0,842 (cf. Tabela 1).

Tabela 1 - Consistência Interna da Escala de dificuldades na amamentação

\begin{tabular}{|c|c|c|c|c|c|c|}
\hline & Itens & Media & $\begin{array}{l}\text { Desvio } \\
\text { Padrão }\end{array}$ & $\begin{array}{l}\text { Correlação } \\
\text { Item/total }\end{array}$ & $\mathbf{R}^{2}$ & $\alpha$ \\
\hline 1 & $\begin{array}{l}\text { Posicionar o bebé para } \\
\text { mamar }\end{array}$ & 2,03 & 0,949 & 0,629 & 0,536 & 0,913 \\
\hline 2 & $\begin{array}{l}\text { Estabelecer uma boa pega do } \\
\text { bebé à mama }\end{array}$ & 2,23 & 1,072 & 0,751 & 0,790 & 0,909 \\
\hline 3 & $\begin{array}{l}\text { Estabelecer uma boa sucção } \\
\text { do bebé à mama }\end{array}$ & 2,23 & 1,070 & 0,733 & 0,753 & 0,909 \\
\hline 4 & Manter uma mamada sem dor & 2,46 & 1,129 & 0,697 & 0,653 & 0,911 \\
\hline 5 & $\begin{array}{l}\text { Manter uma mamada sem } \\
\text { calor e/ou vermelhidão na } \\
\text { mama }\end{array}$ & 2,24 & 1,070 & 0,679 & 0,635 & 0,911 \\
\hline 6 & Manter o mamilo sem ferida & 2,39 & 1,152 & 0,643 & 0,621 & 0,913 \\
\hline 7 & $\begin{array}{l}\text { Fazer o esvaziamento das } \\
\text { mamas }\end{array}$ & 2,26 & 1,090 & 0,641 & 0,452 & 0,913 \\
\hline 8 & $\begin{array}{l}\text { Satisfazer o bebé com o meu } \\
\text { leite }\end{array}$ & 2,11 & 1,216 & 0,693 & 0,617 & 0,911 \\
\hline 9 & $\begin{array}{l}\text { Apresentar mamilos salientes } \\
\text { e adequados à pega }\end{array}$ & 2,08 & 1,182 & 0,638 & 0,459 & 0,913 \\
\hline 10 & $\begin{array}{l}\text { Ter leite em quantidade } \\
\text { suficiente }\end{array}$ & 2,08 & 1,229 & 0,597 & 0,567 & 0,915 \\
\hline 11 & $\begin{array}{l}\text { Manter o bebé desperto } \\
\text { durante a mamada }\end{array}$ & 2,28 & 1,033 & 0,570 & 0,464 & 0,915 \\
\hline 12 & $\begin{array}{l}\text { Manter o bebé interessado na } \\
\text { mama }\end{array}$ & 2,02 & 1,019 & 0,670 & 0,586 & 0,912 \\
\hline 13 & $\begin{array}{l}\text { Receber apoio adequado para } \\
\text { amamentar }\end{array}$ & 1,92 & 0,997 & 0,547 & 0,315 & 0,916 \\
\hline \multirow{2}{*}{\multicolumn{4}{|c|}{ Coeficiente Split-half }} & \multicolumn{3}{|c|}{ Primeira metade $=0,895$} \\
\hline & & & & \multicolumn{3}{|c|}{ Segunda metade $=0,842$} \\
\hline \multicolumn{4}{|c|}{ Coeficiente alpha Cronbach global } & \multicolumn{3}{|l|}{0,919} \\
\hline
\end{tabular}

Avaliamos a adequação da análise factorial exploratória através do teste Kaiser-Meyer-0lkin (KMO). Os valores de referência situam-se entre 0,5 e 1 sendo que no nosso estudo o resultado foi de (KM0=0,893) 0 que per- 


\section{ESCALA DIFICULDADES NA AMAMENTAÇÃo}

mite classificá-lo como bem adequado. Já o teste de esfericidade Bartlett's que se baseia na distribuição estatística de "chi quadrado" testa a hipótese de que não há correlação entre as variaveis não foi avaliado, uma vez que em grandes amostras leva à rejeição da hipótese nula. A solução fatorial, permitiu a extração de 3 fatores que no seu conjunto explicam 68,63\% da variância total.

0 fator 1 designou-se por condições técnicas, explica 23,64\% da variância total e ficou constituído pelos itens 1, 2, e 3, o fator 2 comporta os itens 4, 5, 6 e 7, explica 22,74\% da variância e designou-se por condições maternas, 0 fator 3 explica 22,25\% da variância designou-se por condições do recém-nascido e contém os itens $8,9,10,11,12$ e 13 .

A tabela 2 apresenta a estrutura factorial e as comunalidades $\left(h^{2}\right)$ que deve ser superior a 0,40 (Marôco (2021). Apenas 0 item 13 tem comunalidade inferior mas decidimos pela sua manutenção dada a pertinência para a mensuração do constructo

Tabela 2 - Estrutura fatorial da escala de dificuldades da amamentação

\begin{tabular}{l|l|l|l|l|l}
\hline & Itens & $\begin{array}{l}\text { Fator } \\
\mathbf{1}\end{array}$ & $\begin{array}{l}\text { Fator } \\
\mathbf{2}\end{array}$ & Fator 3 & $\mathbf{h}^{\mathbf{2}}$ \\
\hline 1 & Posicionar o bebé para mamar & 0,800 & & & 0,723 \\
\hline 2 & $\begin{array}{l}\text { Estabelecer uma boa pega do bebé à } \\
\text { mama }\end{array}$ & 0,838 & & & 0,859 \\
\hline 3 & $\begin{array}{l}\text { Estabelecer uma boa sucção do bebé à } \\
\text { mama }\end{array}$ & 0,800 & & & 0,816 \\
\hline 4 & Manter uma mamada sem dor & & 0,819 & & 0,791 \\
\hline 5 & $\begin{array}{l}\text { Manter uma mamada sem calor e/ou } \\
\text { vermelhidão na mama }\end{array}$ & 0,831 & & 0,787 \\
\hline 6 & Manter o mamilo sem ferida & 0,860 & & 0,804 \\
\hline 7 & Fazer o esvaziamento das mamas & & 0,579 & & 0,546 \\
\hline 8 & Satisfazer o bebé com o meu leite & & & 0,782 & 0,748 \\
\hline 9 & $\begin{array}{l}\text { Apresentar mamilos salientes } \\
\text { adequados à pega }\end{array}$ & & & 0,477 & 0,498 \\
\hline 10 & Ter leite em quantidade suficiente & & & 0,864 & 0,790 \\
\hline 11 & Manter o bebé desperto durante a mamada & & & 0,502 & 0,480 \\
\hline 12 & Manter o bebé interessado na mama & & & 0,665 & 0,687 \\
\hline 13 & Receber apoio adequado para amamentar & & & 0,472 & 0,395 \\
\hline Factores & $\%$ Variância & \multicolumn{2}{c}{$\%$ Variância } \\
\hline
\end{tabular}

A solução trifatorial desta escala foi testada através da análise fatorial confirmatória. Analisaram-se pormenores relativos à multicolinearidade e valores univariados e multivariados que pudessem influenciar a análise fatorial. Registamos que as trajetórias dos itens com os fatores a que lhe correspondem são estatisticamente significativos com pesos fatoriais elevados ( $\geq 0,50$ )

Pela figura 1 observa-se que os pesos fatorias se situam acima de 0,50 e a fiabilidade individual é adequada $\left(r^{2} \geq 0,25\right)$ nas três subescalas, 0 que demostra a relevância do fator para predizer os itens.

Os índices de bondade de ajustamento global da AFC apresenta um ajuste pouco adequado para $2 / \mathrm{g} . \mathrm{l}$. = 7,567 e RMSEA $=0,099$ e adequado para $0 \mathrm{GFI}=0,903, \mathrm{CFI}=0,924 ; \mathrm{RMR}=0,071$ e $\mathrm{SRMR}=0,058$. 
Figura 1 - Modelo 1 - Modelo trifatorial inicial da escala de dificuldades de amamentação

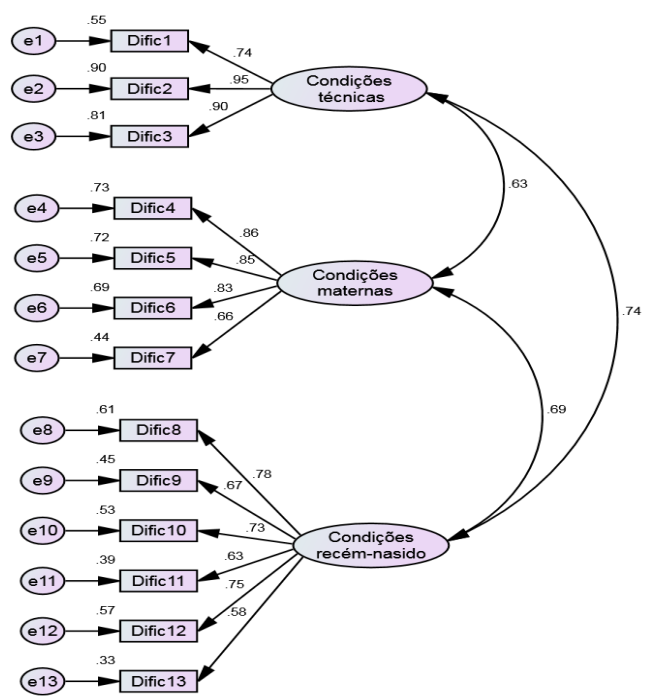

Refinado o modelo com base nos índices de modificação propostos pelo Amos, correlacionando alguns erros regista-se que 0 ajustamento na sua globalidade passou a ser adequado para todos os índicesmas 0 item 11 foi eliminado por apresentar problemas de multicolinearidade.

A figura 2 apresenta o modelo final refinado registando-se uma ligeira melhoria dos índices de bondade de ajustamento global: 2/g.l. = 3,829; $\mathrm{GFI}=0,962 ; \mathrm{CFI}=0,974 ; \mathrm{RMSEA}=0,065 ; \mathrm{RMR}=0,049 ; \mathrm{SRMR}=0,042$.

Figura 2 - Modelo final refinado

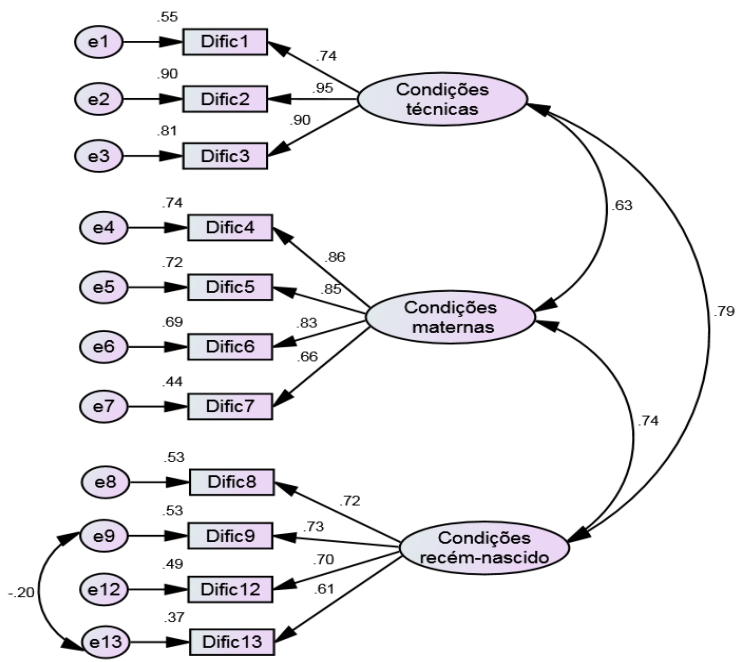




\section{ESCALA DIFICULDADES NA AMAMENTAÇÃo}

Propôs-se uma estrutura hierárquica com um fator de $2^{2}$ ordem desigando por dificuldades de amamentação. A figura 3 ilustra o modelo obtido. Não se registou alteração aos valores de ajustamento global

Figuera 3 - Modelo de $2^{\underline{a}}$ ordem

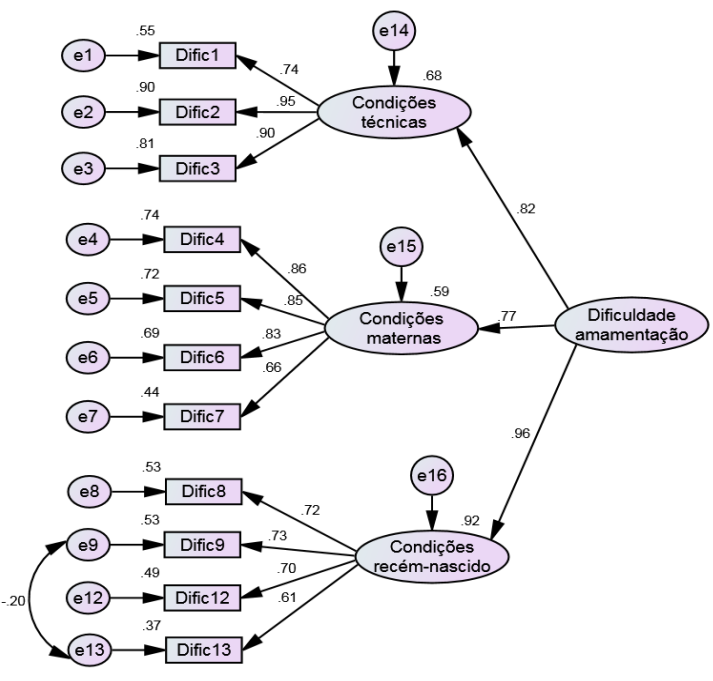

No quadro 1 é apresentada a FC, a VEM, e a VD. A FC é em todos os fatores superiores ao valor de referência $(0,70)$. A VEM indica-nos que o fator três tem um valor inferior ao recomendado. Não é patente a existência de VD entre os fator 2 e 0 fator 3 pois os valores correlacionais são superiores à VEM. Acrescenta-se que 0 coeficiente estratificado é elevado $(0,945)$ com uma VEM de 0,596.

Quadro 1 - Fiabilidade compósita e Variância extraída média da escala de dificuldades na amamentação

\begin{tabular}{|l|l|l|l|l|l|}
\hline \multirow{2}{*}{ Fatores } & \multirow{2}{*}{ F C } & \multirow{4}{*}{ VEM } & \multicolumn{3}{|l|}{$\begin{array}{l}\text { Validade } \\
\text { descriminante }\end{array}$} \\
\cline { 5 - 8 } & & & F2 & F3 & F2 \\
\hline F1 - Condições técnicas & 0,900 & 0,752 & 0,396 & 0,624 & \\
\hline F2 - Condições maternas & 0,878 & 0,646 & & 0,547 & \\
\hline F3- Condições recém-nascido & 0,810 & 0,462 & & & \\
\hline
\end{tabular}

$\mathrm{FC}$ estatificado $=0,945 \mathrm{VEM}=0,596$

Terminamos o estudo da escala, com referência ao estudo de consistência interna por subescala dos itens remanescentes.

No Fator 1 - Condições técnicas, os coeficientes de alpha de Cronbach dão indicações de razoável e muito boa consistência interna, sendo o menor valor $(=0,783)$ encontrado no item 2 e o maior $(=0,921)$ no item 1 .

No Fator 2 - Condições maternas, os coeficientes de alpha de Cronbach revelam boa consistência interna, sendo o menor valor $(=0,820)$ registados nos itens 5 e 6 e o maior $(=0,886)$ no item 7.

No Fator 3 - Condições do recém-nascido, os coeficientes de alpha de Cronbach dão indicações de razoável consistência interna, sendo o menor valor $(=0,680)$ o do item 8 e 0 maior $(=0,757)$ no item 13 , com um alfa total de 0,773 (cf. tabela 3). 
Tabela 3 - Consistência interna por subescalas da Escala de Dificuldades na Amamentação

\begin{tabular}{|c|c|c|c|c|c|c|}
\hline Itens & & Média & Dp & $\begin{array}{l}\text { r/item } \\
\text { total }\end{array}$ & $\mathbf{r}^{2}$ & $\begin{array}{l}\alpha \text { sem } \\
\text { item }\end{array}$ \\
\hline & Condições Técnicas & & & & & $\mathbf{0 , 8 9 4}$ \\
\hline 1 & Posicionar o bebé para mamar & 2,03 & ,949 & 705 &, 510 & 0,921 \\
\hline 2 & $\begin{array}{l}\begin{array}{l}\text { Estabelecer uma boa pega do } \\
\text { bebé à mama }\end{array} \\
\end{array}$ & 2,23 & 1,072 & 866 & 771 & 0,783 \\
\hline \multirow[t]{2}{*}{3} & $\begin{array}{l}\text { Estabelecer uma boa sucção do } \\
\text { bebé à mama }\end{array}$ & 2,23 & 1,070 & 818 & 732 & 0,826 \\
\hline & Condições maternas & & & & & $\mathbf{0 , 8 7 4}$ \\
\hline 4 & Manter uma mamada sem dor & 2,46 & 1,129 & 0,771 & 0,624 & 0,822 \\
\hline 5 & $\begin{array}{l}\text { Manter uma mamada sem calor } \\
\text { e/ou vermelhidão na mama }\end{array}$ & 2,24 & 1,070 & 0,777 & 0,623 & 0,820 \\
\hline 6 & Manter o mamilo sem ferida & 2,39 & 1,152 &, 774 & 611 & 0,820 \\
\hline \multirow[t]{2}{*}{7} & $\begin{array}{l}\text { Fazer o esvaziamento das } \\
\text { mamas }\end{array}$ & 2,26 & 1,090 & 0,603 & 0,366 & 0,886 \\
\hline & Condições do recém-nascido & & & & & $\mathbf{0 , 7 7 3}$ \\
\hline 8 & $\begin{array}{l}\text { Satisfazer o bebé com o meu } \\
\text { leite }\end{array}$ & 2,11 & 1,216 & 0,644 & 0,418 & 0,680 \\
\hline 9 & $\begin{array}{lll}\begin{array}{l}\text { Ter leite em quantidade } \\
\text { suficiente }\end{array} & \text { en } \\
\end{array}$ & 2,08 & 1,182 & 0,561 & 0,335 & 0,727 \\
\hline 12 & $\begin{array}{l}\text { Manter o bebé interessado na } \\
\text { mama }\end{array}$ & 2,02 & 1,019 & 0,615 & 0,380 & 0,700 \\
\hline 13 & $\begin{array}{l}\text { Receber apoio adequado para } \\
\text { amamentar }\end{array}$ & 1,92 & 0,997 & 0,494 & 0,259 & 0,757 \\
\hline
\end{tabular}

A tabela 4 apresenta a validade convergente/divergente dos itens apurando-se que todos os itens apresentam validade convergente com o fator que lhe corresponde já que o valor correlacional é mais elevado com a subescala a que pertence. 
Tabela 4 - validade convergente /divergente dos itens

\begin{tabular}{l|l|l|l|l|l}
\hline & Itens & $\mathbf{F 1}$ & $\mathbf{F 2}$ & $\mathbf{F 3}$ & Ftotal \\
\hline 1 & Posicionar o bebé para mamar & $0,854^{* *}$ & $0,474^{* *}$ & $0,552^{* *}$ & $0,697^{* *}$ \\
\hline 2 & $\begin{array}{l}\text { Estabelecer uma boa pega do bebé à } \\
\text { mama }\end{array}$ & $0,945^{* *}$ & $0,565^{* *}$ & $0,660^{* *}$ & $0,808^{* *}$ \\
\hline 3 & $\begin{array}{l}\text { Estabelecer uma boa sucção do bebé } \\
\text { à mama }\end{array}$ & $0,924^{* *}$ & $0,573^{* *}$ & $0,642^{* *}$ & $0,798^{* *}$ \\
\hline 4 & Manter uma mamada sem dor & $0,551^{* *}$ & $0,878^{* *}$ & $0,563^{* *}$ & $0,779^{* *}$ \\
\hline 5 & $\begin{array}{l}\text { Manter uma mamada sem calor e/ou } \\
\text { vermelhidão na mama }\end{array}$ & $0,500^{* *}$ & $0,877^{* *}$ & $0,555^{* *}$ & $0,759^{* *}$ \\
\hline 6 & Manter o mamilo sem ferida & $0,447^{* *}$ & $0,882^{* *}$ & $0,515^{* *}$ & $0,728^{* *}$ \\
\hline 7 & Fazer o esvaziamento das mamas & $0,526^{* *}$ & $0,770^{* *}$ & $0,541^{* *}$ & $0,716^{* *}$ \\
\hline 8 & Satisfazer o bebé com o meu leite & $0,522^{* *}$ & $0,536^{* *}$ & $0,828^{* *}$ & $0,725^{* *}$ \\
\hline 9 & $\begin{array}{l}\text { Apresentar mamilos salientes e } \\
\text { adequados à pega }\end{array}$ & $0,565^{* *}$ & $0,517^{* *}$ & $0,774^{* *}$ & $0,709^{* *}$ \\
\hline 12 & Manter o bebé interessado na mama & $0,577^{* *}$ & $0,453^{* *}$ & $0,783^{* *}$ & $0,689^{* *}$ \\
\hline 13 & $\begin{array}{l}\text { Receber apoio adequado para } \\
\text { amamentar }\end{array}$ & $0,441^{* *}$ & $0,455^{* *}$ & $0,698^{* *}$ & $0,613^{* *}$ \\
\hline
\end{tabular}

Por fim apresenta-se a matriz de correlações entre as diferentes subescalas com valores de correlação moderados a elevados positivos e significativos com 0 factor total da escala com percentagens de variância explicada acima de 35,0\% entre factores e de $71 \%$ com o factor global (cf. tabela 5).

Tabela 5 - Matriz de Correlação de Pearson entre os fatores da Escala de Dificuldades no Aleitamento Materno

\begin{tabular}{l|l|l|l|l}
\hline Fatores & $\begin{array}{l}\text { Condições } \\
\text { Técnicas }\end{array}$ & $\begin{array}{l}\text { Condições } \\
\text { maternas }\end{array}$ & $\begin{array}{l}\text { Condições } \\
\text { do recém- } \\
\text { nascido }\end{array}$ & $\begin{array}{l}\text { Fator } \\
\text { global }\end{array}$ \\
\hline Condições Técnicas & - & $0,593^{* *}$ & $0,682^{* *}$ & $0,847^{* *}$ \\
\hline Condições maternas & $0,593^{* *}$ & - & $0,637^{* *}$ & $0,875^{* *}$ \\
\hline $\begin{array}{l}\text { Condições do recém- } \\
\text { nascido }\end{array}$ & $0,682^{* *}$ & $0,673^{* *}$ & - & $0,888^{* *}$ \\
\hline Fator global & $0,847^{* *}$ & $0,875^{* *}$ & $0,888^{* *}$ & - \\
\hline \multicolumn{4}{l}{$\mathrm{p}>0,001$} &
\end{tabular}

\section{DISCUSSÃO/CONCLUSÃO}

Os resultados evidenciam que a escala de dificuldades da amamentação apresenta uma estrutura fatorial com resultados satisfatórios de validade e de confiabilidade, representando adequadamente os constructos em questão. A escala de dificuldades de amamentação pode ser utilizada como instrumento de pesquisa para avaliar as dificuldades sentidas pelas puérperas no processo de amamentação, no que se refere às condições técnicas, maternas ou do recém-nascido.

Assim, deve considerar-se que 0 abandono do aleitamento materno até aos 6 meses resulta essencialmente por dificuldades da amamentação, sobretudo no que respeita às condições técnicas e às condições do recémnascido. Alguns estudos revelam que as mães relatam dificuldades na técnica da pega (Dias et al. 2013, p. 313). Também Castelli et al. (2015, p. 1180) constaram que, num universo de 69\% das queixas apresentadas, estas se relacionaram com a pega do recém-nascido $(27,6 \%)$, a dor nas mamas $(27,6 \%)$ e com as fissuras mamárias $(13,8 \%)$. 
0 estudo de revisão sistemática de Karaçam and Sa lık (2018), concluiu que as mulheres desistiram da amamentação devido à presença de problemas (24,5\%), deficiência de leite materno / preocupação com a deficiência de leite / pensar que o bebê não está satisfeito / ganho de peso inadequado do bebé (15,7\%), falta de conhecimento e experiência sobre amamentação / necessidade de educação e apoio (17,8\%). Referem ainda que as mulheres relataram problemas com mamilo plano / deprimido / pequeno $(7,7 \%)$, dor / sensibilidade $(3,9 \%)$, edema / tumefação / ingurgitamento (10,8\%), vermelhidão (28,8\%), rachadura / ferida / sangramento (26,1\%) e mastite $(5,6 \%)$.

Este estudo tornou evidente que as mulheres registaram ibnumeros problemas com a amamentação e que mais educação / aconselhamento / acompanhamento pré-natal foi uma das estratégias utilizadas para reduzir os problemas.

Também no estudo de Duarte et al. (2019) se verificou que existe uma multiplicidade de fatores que influencia a motivação, a prática e manutenção do aleitamento materno, pelo que os autores recomendam programas que promovam a manutenção e a existência de apoio por parte dos profissionais de saúde para ajudar a superar as dificuldades encontradas, prevenindo 0 abandono precoce do aleitamento materno.

Em conclusão, as dificuldades na amamentação, quer sejam relacionadas à técnica, às condições maternas ou do recém-nascido devem ser tidas em consideração por parte dos enfermeiros, mas também da população em geral, sendo que a disponibilização de uma escala que melhor permita essa mesma identificação é encarada como uma mais valia na prática de cuidados.

Nesse sentido, o instrumento parece revelar-se adequado nesta amostra, pelo que poderá constituir-se como um recurso valioso para o estudo das dificuldades de amamentação na população portuguesa.

\section{AGRADECIMENTOS}

Este trabalho é financiado por Fundos Nacionais através da FCT - Fundação para a Ciência e a Tecnologia, I.P., no âmbito do projeto Ref ${ }^{\mathrm{a}}$ UIDB/00742/2020. Agradecemos adicionalmente à Unidade de Investigação em Ciências da Saúde: Enfermagem (UICISA: E) e ao Politécnico de Viseu pelo apoio prestado.

Agradecemos também a todos os envolvidos no projeto de investigação "Prevalência da Amamentação. Motivação, dificuldades e a ajuda dos enfermeiros" nomeadamente aos estudantes: Ana Cristina Pereira, Cristiana Machado, Daniel Coelho, Daniela Martins, Ana Solange Bastos, Diana Albino, Dulce Figueiral, Patrícia Taveira; e aos profesores: João Carvalho Duarte (mentor do tratamento estatístico), Odete Amaral, Rui Dionísio.

\section{REFERENCIAS BIBLIOGRÁFICAS}

Castelli, C., Maahs, M., \& Almeida, S. (2014). Identificação das dúvidas e dificuldades de gestantes e puérperas em relação ao aleitamento materno. Rev. CEFAC; 16(4): 1178-1186. Acedido em http://www.scielo.br/pdt/rcefac/v16n4/1982-0216-rcefac-16-4-1178.pdf

Damásio, B. F. (2012). Uso da análise fatorial exploratória em psicologia. Avaliação Psicológica, 11, 213-228. Retrieved from http://pepsic.bvsalud.org/scielo.php?script=sci_arttext\&pid=S1677-04712012000200007\&nrm=iso

Dias, A., Monteiro, T., Oliveira, D., Guedes, A., Godinho, C., \& Alexandrino, A.M. (2013). Aleitamento materno no primeiro ano de vida: prevalência, fatores protetores e de abandono. Acta Pediátrica Portuguesa. Sociedade Portuguesa de Pediatria, 44(6), 313-318. Acedido em http://actapediatrica.spp.pt/article/view/2719/2754

Duarte, J., Nelas, P., Coutinho, E., Chaves, C., Amaral, O., \& Dionísio, R. (2019). Influência das características obstétricas e maternas na prevalência do aleitamento materno. International Journal of Developmental and Educational Psychology, Revista INFAD de Psicologia, 4(1), 10. doi:10.17060/ijodaep.2019.n1.v4.1574

Karaçam, Z., \& Sa lık, M. (2018). Breastfeeding problems and interventions performed on problems: systematic review based on studies made in Turkey. Turk pediatri arsivi, 53(3), 134-148.

doi:https://doi.org/10.5152/TurkPediatriArs.2018.6350 


\section{ESCALA DIFICULDADES NA AMAMENTAÇÃo}

Karaçam, Z., \& Sa lık, M. (2018). Breastfeeding problems and interventions performed on problems: systematic review based on studies made in Turkey. Turk pediatri arsivi, 53(3), 134-148. https://doi.org/10.5152/TurkPediatriArs.2018.6350

Marôco, J. (2021). Análise estatística com SPSS Statistics (ReportNumber Ed. 8 ed.): ReportNumber.

Office of the Surgeon General (US), Centers for Disease Control and Prevention (US), Office on Women's Health (US), The Surgeon General's Call to Action to Support Breastfeeding. Rockville (MD), \& Office of the Surgeon General (US). (2011). Barriers to Breastfeeding in the United States. Retrieved from https://www.ncbi.nlm.nih.gov/books/NBK52688/

Oliveira, M. (2016). Aleitamento materno: Estudo de prevalência e fatores condicionantes nos primeiros seis meses de vida. Pensar Enfermagem, 20(1), 4-15. Retrieved from http://pensarenfermagem.esel.pt/files/Artig01_4_15.pdf

Pasquali, L. (2003). Psicometria: Teoria dos Testes na Psicologia e na Educação. Petrópolis: Vozes

Pestana, M., \& Gageiro, J. (2014). Análise de dados para ciências sociais: a complementaridade do SPSS (6ª ed. ed.). Lisboa: Edições Sílabo.

Rasteiro, R., Santos, E., \& Coutinho, E. (2021). Necessidades e Preocupações Maternas no Período Pós-Parto: Revisão Sistemática da Literatura. New Trends in Qualitative Research, 8, 817-827. doi:https://doi.org/10.36367/ntqr.8.2021.817-827

WABA. (2021). World Breastfeeding Week 2021. Protect Breastfeeding: A Shared Responsibility. Retrieved from https://worldbreastfeedingweek.org/

Walker, M. (2008). Conquering common breast-feeding problems. J Perinat Neonatal Nurs, Oct-Dec;22(4), 267274. doi:10.1097/01.JPN.0000341356.45446.23. PMID: 19011490

WHO, \& UNICEF. (2021). Joint statement by UNICEF Executive Director Henrietta Fore and WHO Director-General Dr. Tedros Adhanom Ghebreyesus on the occasion of World Breastfeeding Week. Retrieved from https://www.who.int/news/item/01-08-2021-joint-statement-by-unicef-executive-director-henrietta-foreand-who-director-general-dr.-tedros-adhanom-ghebreyesus-on-the-occasion-of-world-breastfeeding-week 